\title{
Échec scolaire et démocratisation: enjeux, réalités, concepts, problématiques et résultats de recherche
}

\section{Jean-Yves Rochex}

Les notions d'échec scolaire et de démocratisation du système éducatif et de l'accès $a u(x)$ savoir $(x)$ sont à la fois étroitement liées et éminemment problématiques. Polysémiques, elles désignent des réalités contradictoirs, que l'analyse se doit évidemment de distinguer mais perd beaucoup à opposer. Mieux comprendre, pour mieux agir, requiert dès lors de travailler à dépasser le risque d'un partage du travail entre une sociologie des inégalités scolaires insuffisamment soucieuse des modalités effectives des activités de transmission et d'appropriation des savoirs et techniques intellectuelles, et une recherche en pédagogie et en psychologie des apprentissages insuffisamment soucieuse des différents contextes sociaux, institutionnels et didactiques dans lesquels ces activités sont toujours situées. Ce qui nous demande de savoir établir des rapports renouvelés entre sociologie, psychologie et didactique.

Que ce soit dans les travaux de recherche ou dans les débats sociaux et politiques concernant l'École et les politiques éducatives - débats plus vifs et passionnés en France que dans la plupart des pays comparables -, les thématiques de «l'échec scolaire» et de la démocratisation du système éducatif et de l'accès $\mathrm{au}(\mathrm{x})$ savoir $(\mathrm{s})$ et à la formation sont à la fois étroitement liées et éminemment problématiques. Ainsi les débats et polémiques sur la persistance ou le recul de l'échec scolaire et sur son ampleur, sur la nature, les modalités et les insuffisances de la démocratisation de notre système éducatif, ou sur son caractère fictif, sont-ils liés aux niveaux de formation et aux acquisitions auxquels on s'intéresse, aux outils statistiques et aux instruments de mesure que l'on utilise, à l'empan temporel que l'on prend en considération, et aux définitions et aux conceptions de l'échec ou de la réussite scolaires et de la démocratisation qui sous-tendent, sans pour autant être toujours explicitées, telle ou telle appréciation ou prise de position. 


\section{Des notions polysémiques et des réalités contradictoires}

Dans un texte devenu classique, Viviane Isambert-Jamati a montré comment, dans un contexte marqué par l'unification du système éducatif et la généralisation progressive de l'accès au secondaire dans les années 60 , l'émergence de la thématique de l'échec scolaire a noué ensemble deux questions jusqu'alors traitées séparément : celle des écarts sociaux de scolarisation, et celle de l'existence d'une fraction, plus ou moins importante, d'élèves considérés comme ne satisfaisant pas aux exigences propres aux situations et aux cursus scolaires dans lesquels ils étaient inscrits (Isambert-Jamati, 1985). D'où la double acception du terme "échec scolaire»: acception relationnelle ou relative, tout d'abord, renvoyant au fait pour un élève ou une catégorie d'élèves d'occuper les rangs inférieurs dans la hiérarchie des positions scolaires et d'être ainsi plus particulièrement affectés par les conséquences négatives qui en découlent en matière d'orientation, de qualification, d'accès à l'emploi, de conditions d'insertion socio-professionnelle; acception spécifique ou «absolue», renvoyant au fait pour un élève ou une catégorie d'élèves de réaliser, ou d'échouer à réaliser, tel apprentissage ou telle acquisition cognitive et culturelle (que l'on pense aux débats, inépuisables faute de critères communs, qui reviennent régulièrement dans les salles de professeurs et les médias sur «'illettrisme» des jeunes ou sur la proportion d'élèves qui ne «sauraient pas lire» à l'entrée au collège), d'atteindre ou non tel «niveau» (lequel est parfois bien difficile à définir) de culture scolaire, de formation, de scolarisation ou de qualification. Nombre d'incompréhensions et de faux débats, mais aussi de paradoxes propres à la thématique de l'échec scolaire sont dus au fait que les réalités auxquelles renvoient l'une et l'autre de ces deux acceptions évoluent de façon relativement différente, en fonction de logiques relativement indépendantes. Seule la prise en considération de ces réalités différenciées peut permettre de rendre compte du contraste entre l'élévation considérable du niveau de formation des nouvelles générations qu'a connue le système éducatif français depuis quarante ans, avec la généralisation de l'accès au collège de 1960 à 1975, puis la massification de l'accès au lycée de 1985 à 1995, et l'émergence, la persistance, voire l'exacerbation du débat social, médiatique et pédagogique sur «l'échec scolaire» et les phénomènes sociaux qui lui sont associés. De même la tonalité, optimiste ou pessimiste, des propos de tel observateur ou analyste, s'explique-t-elle bien souvent par le type de données pris en considération, les uns mettant en valeur l'élévation du niveau moyen de formation tandis que les autres insisteront plus sur la permanence, depuis 1994, d'un «seuil» de 8 à $9 \%$ d'une classe d'âge (environ 60000 jeunes) sortant du système éducatif sans qualification, et donc sur l'incapacité de notre institution scolaire à poursuivre le progrès spectaculaire qui avait vu ce taux passer de plus de $40 \%$ au début des années 60 à $20 \%$ en 1976, puis à $10 \%$ en 1990 (Broccolichi et Larguèze, 1996; Éducation et Formations, 2000). 
Le terme "démocratisation» recouvre, lui aussi, plusieurs acceptions, que l'analyse se doit de distinguer mais perd beaucoup à opposer l'une à l'autre. La première, souvent appelée "démocratisation quantitative», est particulièrement attentive à l'évolution des taux d'accès de l'ensemble des classes d'âge successives et des différents groupes sociaux qui les composent aux différents niveaux de scolarisation et de certification; la seconde, souvent nommée «démocratisation qualitative», s'attache plus particulièrement à l'étude et à l'évaluation des inégalités entre catégories sociales et de leur évolution (Prost, 1986, 2001; Rochex, 1991). Ainsi, au premier sens du terme, il y a bien eu démocratisation de notre système éducatif, liée à la généralisation de l'accès au premier cycle du secondaire dans les années 1960-1970, puis à l'élargissement considérable de l'accès au lycée et aux différents types de baccalauréat, depuis le milieu des années 1980: de nombreux jeunes, issus dans leur grande majorité des classes moyennes et populaires, ont ainsi accédé à des formations et des diplômes, à des savoirs et des compétences, auxquels leurs parents ou leurs aînés n'avaient pu accéder, voire n'auraient même pas imaginé pouvoir accéder. En revanche, force est de constater que les inégalités entre catégories sociales ne se sont pas ou guère réduites, qu'elles n'ont, pour l'essentiel, fait que se déplacer vers l'aval, l'accès au lycée, voire à l'enseignement post-bac jouant aujourd'hui le rôle différenciateur que jouait l'accès au premier cycle de l'enseignement secondaire il y a quelques décennies: en ce deuxième sens, il n'y a guère eu démocratisation ${ }^{1}$. Mais il est nécessaire de ne pas choisir entre chacun de ces constats pour pouvoir comprendre que, si les inégalités sociales en matière de scolarisation et d'accès au savoir demeurent très fortes, les modalités selon lesquelles elles sont produites ont, elles, profondément changé, ainsi que le rapport des différents groupes sociaux à l'institution et aux enjeux scolaires.

Une mise en perspective historique des inégalités scolaires en France (DuruBellat et Kieffer, 1999) montre que la démocratisation par l'ouverture de l'accès au collège, puis au lycée, a été fortement contrariée par le maintien, voire l'accroissement des taux de sélectivité interne propres à ces niveaux de scolarisation. Ainsi, dans les décennies 1960-70, le collège n'est en rien devenu moins sélectif au fur et à mesure que les enfants d'origine populaire y entraient plus largement, la sélectivité passant par les orientations précoces vers l'enseignement professionnel court qui a dès lors été de plus en plus perçu comme filière de relégation. Les évolutions structurelles et politiques ayant conduit à supprimer, pour une large part, les orientations précoces avant la quatrième et dernière année du collège, ce processus s'est décalé vers l'aval, et l'ouverture de l'accès au second cycle secondaire est aujourd'hui contrariée par l'orientation dans les différentes filières d'enseignement général ou technologique, dont le recrutement est dès lors de plus en plus socialement différencié. Au lycée, si les filières scientifiques générales n’ont connu qu'une démocratisation toute relative, les filières conduisant en trois ans après le collège à un baccalauréat technologique, en particulier les filières tertiaires, se sont, elles, fortement "prolétarisées», au point d'être aujourd'hui per- 
çues comme filières de relégation au même titre que l'enseignement professionnel court (conduisant à un diplôme d'ouvrier ou employé qualifié en deux ans après la deuxième ou la quatrième classe de collège) il y a deux ou trois décennies. L'ensemble de ce processus a ainsi pu être caractérisée comme mouvement de «démocratisation ségrégative» (Merle, 2000).

Pour autant, ces filières dévalorisées ne sauraient être considérées unilatéralement comme n'étant que filières de relégation. En s'élargissant et en se diversifiant par la création de filières technologiques, puis professionnelles, l'offre scolaire secondaire a produit un important «effet d'offre» (Galland, 1988) permettant à de nombreux jeunes, particulièrement d'origine populaire, d'obtenir un baccalauréat alors qu'ils avaient ou auraient été exclus des filières d'enseignement général, et de renouer, une fois leur diplôme obtenu, avec des ambitions et des aspirations de poursuite d'études auxquelles ils avaient cru devoir renoncer suite aux vicissitudes de leur orientation. Ce processus a contribué à amplifier la demande sociale de scolarisation, parallèlement exacerbée par la dégradation considérable et durable des conditions d'insertion socio-professionnelle des jeunes sortants du système éducatif (chômage, précarité, divorce formation-emploi...), demande sociale qui nourrit aussi bien les stratégies familiales consuméristes de recherche de la «bonne école» (Ballion, 1991) ou d'évitement des écoles ou établissements jugés difficiles (Broccolichi et van Zanten, 1997) que les mouvements de protestation sociale qui ont marqué lors des trois dernières années différents départements français (Seine-Saint-Denis, Gard, Hérault, Guyane...) particulièrement défavorisés dans le domaine scolaire.

On voit à travers ce trop bref résumé combien la progression réelle des scolarités et l'élévation considérable du niveau de formation des jeunes générations se sont conjuguées avec le maintien et le renouvellement des processus de différenciation sociale, et avec une forte dégradation des conditions d'insertion socioprofessionnelles. Le doute a ainsi été jeté sur le sens de l'École, de ce qu'on y apprend et de ce que l'on peut en espérer, sur le sens des mobilisations personnelles et familiales qu'elle requiert (Beaud et Pialoux, 1999; Charlot, Bautier et Rochex, 1992; de Queiroz, 1981, 1991; Dubet, 1991; Montandon et Perrenoud, 1987; Rayou, 1998; Rochex, 1995; Terrail, 1997; Thin, 1998). Mais si l'élévation générale des niveaux de formation nourrit, voire exacerbe les différences et les ségrégations, en retour, l'expérience et l'ampleur des inégalités réelles nourrit et attise les aspirations et les revendications égalitaires: telle est la contradiction structurelle majeure du système éducatif français, que nous invitent et nous contraignent à penser le double sens du terme "démocratisation» et les données statistiques qu'il recouvre.

Reste que la réflexion sur les inégalités sociales et la démocratisation de l'accès au savoir ne peut se limiter à l'évaluation des taux d'accès des différentes catégories sociales aux différents niveaux de scolarisation sans se poser également la question des conditions d'étude et d'apprentissage et de l'effectivité des acquisitions intellectuelles des élèves. De ce point de vue, s'il est nécessaire de dire que 
le discours de déploration sur la «baisse du niveau» est faux et mensonger (Baudelot et Establet, 1989), on peut néanmoins penser que le rapport entre niveau de scolarisation (voire de certification) et niveau d'acquisitions effectivement réalisées est de plus en plus problématique, et qu'il est source d'inégalités croissantes entre établissements et entre groupes sociaux. De fait, la ségrégation urbaine, mais aussi les logiques parentales consuméristes et la mise en concurrence accrue des établissements conduisent à un accroissement relativement masqué des inégalités et à une homogénéisation sociale croissante du recrutement des différents collèges et lycées, en particulier dans les quartiers urbains populaires. La concentration des difficultés mais aussi les modes d'adaptation des enseignants et des établissements aux caractéristiques et aux difficultés, réelles ou supposées, des élèves peuvent conduire à minorer les exigences et le temps consacré aux apprentissages - par exemple au profit d'actions ou projets dits «de socialisation» - et à redoubler les différences de recrutement entre établissements par des différences d'exigences, souvent insues y compris de ceux qui les mettent en œuvre (Bautier et Rochex, 1997; Bouveau et Rochex, 1997; Broccolichi, 1995). On touche ici à une autre manière, tout aussi nécessaire que celles précédemment évoquées, de penser et de poser les questions de la démocratisation et des inégalités.

Plus grave encore peut-être, les modes de gestion de la difficulté scolaire se sont, ces dernières années, plus préoccupés de gestion des flux, de passage ou d'orientation, que de l'effectivité des acquisitions cognitives en principe requises pour accéder à tel ou tel niveau d'enseignement, le rapport entre la «carrière» ou le cheminement des élèves et les apprentissages réalisés devenant ainsi de plus en plus problématique. En témoignent les données statistiques suivantes. Premièrement, une comparaison des performances des élèves de la dernière année d'école primaire, évaluées en 1987 et 1997 à l'aide des mêmes épreuves et des mêmes protocoles, montre une très grande stabilité tant des performances moyennes que de leur dispersion, ce alors que l'on observe dans le même temps une évolution importante de la structure d'âge des populations concernés, due aux mesures de restriction des redoublements: la proportion d'élèves «à l'heure» est passée en dix ans de 64 à $77 \%$, la proportion d'élèves «en retard» diminuant d'autant, sans donc que les performances évaluées aient connu de progression. Un tel divorce concerne en particulier les $10 \%$ d'élèves les plus faibles (qui sont majoritairement des garçons issus de milieux populaires), dont les résultats demeurent eux aussi très stables, mais qui sont néanmoins admis en plus grande proportion à entrer au collège en 1997 (82,4 \%) que dix années auparavant (76,5\%) (Baktavatsalou et Pons, 1998). Deuxièmement, l'étude des parcours suivis par les entrants au collège en 1989 et en 1995 montre que, si la proportion d'élèves qui ne redoublent pas durant les trois premières années du cursus progresse de trois points, ce "progrès» est exclusivement du aux élèves les plus en difficulté. Ainsi les élèves entrés au collège avec deux ans de retard gagnent-ils près de six points, et ils redoublent moins que leurs pairs entrés avec seulement un an de retard. Ce 
phénomène, qui ressemble plus à une éviction par le haut qu'à une réelle progression du niveau des acquisitions des élèves concernés est beaucoup plus accentué dans les collèges situés en Zone d'éducation prioritaire que dans les autres (Poncet, 2000). Sans pour autant penser que le retour à la pratique du redoublement serait une meilleure solution, on peut estimer, à la lumière des travaux plus qualitatifs précédemment évoqués, qu'une telle politique de «fuite en avant» ne peut que rendre les inégalités scolaires encore plus opaques pour les familles les plus démunies, et être source, pour les enseignants exerçant dans les écoles et les établissements les plus concernés, d'un désarroi profond sur le sens que l'on veut et qu'eux-mêmes peuvent donner à leur métier.

\section{Les pratiques de savoir: objet suspect pour la sociologie de l'éducation?}

Il n'est guère original de rappeler ici que la sociologie de l'École et des inégalités scolaires a connu en France d'importantes évolutions depuis les grandes synthèses statistiques et macro-sociologiques des années 1960-70. Les principales de ces évolutions - dont les liens sont évidents mais qui ne sont pas pour autant identifiables les unes aux autres - sont sans doute: l'ouverture de ces «quasi boîtes noires» que demeuraient la classe, l'établissement ou la communauté (Henriotvan Zanten, Derouet et Sirota, 1987a et b); l'objectif d'étudier «les inégalités en train de se faire» et leurs processus de production plutôt que de postuler qu'elles seraient toujours «jouées d'avance»; le déplacement d'une posture de critique, voire de dénonciation, globale de l'institution scolaire, de sa fonction et de ses modes de fonctionnement, à une préoccupation d'amélioration (démocratisation et plus grande efficacité) de cette institution et une volonté de mise en évidence de marges d'action chez ses agents et protagonistes; enfin la relative perte de faveur des paradigmes macro-sociologiques (fonctionnalistes ou conflictualistes) au profit des approches et des paradigmes constructivistes inspirés de la phénoménologie sociale, de l'interactionnisme symbolique et de l'ethnométhodologie (sur ces évolutions, cf. entre autres Barrère et Sembel, 1998; Duru-Bellat, 2000; Duru-Bellat et van Zanten, 1999; de Queiroz, 1995).

Les différentes recherches au travers desquelles se sont réalisées ces évolutions se sont penchées sur les représentations sociales, les catégories d'entendement, de jugement, d'évaluation et d'orientation des enseignants, sur les modes de constitution des classes, sur les interactions maîtres-élèves, sur les diverses modalités d'imposition, de négociation ou de transgression des règles et des routines d'un ordre scolaire décrit et pensé beaucoup plus en termes "polémologiques» qu'en termes socio-cognitifs. En revanche, elles ne se sont guère intéressées aux rapports entre ces différents phénomènes et les processus et activités d'enseignement et d'apprentissage de contenus cognitifs et culturels et de pratiques de savoir spécifiés, lesquels obéissent à leurs propres normes, qui sont pour une part irréduc- 
tibles à celles de l'ordre scolaire (ce dont témoigne par exemple le double sens du mot discipline en français). De sorte que, si ces travaux apportent des éclairages tout à fait intéressants sur ce qui se passe et se joue au sein des enceintes scolaires, s'ils posent des questions majeures et redoutables à l'institution scolaire, aux pratiques, aux catégories d'interprétation et aux modes d'interaction mis en œuvre par ses différents agents et protagonistes, ils ne nous apprennent que fort peu de choses sur les processus individuels et sociaux qui permettent, favorisent ou au contraire entravent l'appropriation de contenus et de pratiques de savoir par des élèves appartenant à différents milieux sociaux, sur les modes de traitement de ces contenus et pratiques dans les situations et dans les activités d'enseignement et d'apprentissage des uns et des autres. Au final, et à de rares exceptions près ${ }^{2}$, la fonction de transmission du savoir et ses modalités pratiques, les modes de circulation, d'appropriation et d'étude des savoirs dans et hors l'institution scolaire apparaissent comme étant le parent pauvre de la sociologie de l'éducation: à la fois objet opaque et, dans une certaine mesure, objet suspect, «mauvais objet», comme si la posture du «soupçon» et les visées de «dévoilement» adoptées et mises en œuvre dans leurs recherches par bien des sociologues ne pouvaient que les conduire à considérer et à traiter cette fonction de transmission du savoir comme n'étant qu'illusion, mystification, leurre derrière lesquels se cachent les "véritables fonctions» (reproduction sociale, aliénation, domestication...) de l'institution scolaire dont la sociologie aurait pour objet de rechercher et de mettre à jour les modes opératoires (Charlot et al., 1992; Lahire, 1993, 1999; Rochex, 1993, 1995).

Cette réticence forte de la sociologie de l'éducation à prendre en considération la fonction et les activités de transmission et d'appropriation du savoir, à en ouvrir les boîtes noires après avoir ouvert celles de la classe et de l'établissement, a deux conséquences qui me semblent devoir être objets de débat plus qu'elles ne le sont dans nos milieux de recherche. D'une part, elle conduit à ce que, dans leurs problématiques comme dans leurs objets, nombre de travaux appréhendent la scolarisation comme un cadre, une scène, une arène, où se révèlent, se construisent, s'agencent et se confrontent des processus sociaux, des logiques d'action et d'expérience d'ordre général, plus que comme fonction sociale et systèmes d'activités spécifiques, confrontées à des objets et à des contraintes ayant leur caractère propre qui ne sauraient être réduits à de pures exigences arbitraires et élitistes (Forquin, 1989). Dénuées de toute spécificité ou voyant celle-ci minorée, voire réfutée, par nombre d'analyses et de problématiques de recherche, la scolarisation et les pratiques de savoir qui s'y déroulent (le non-apprentissage ou le refus d'apprentissage pouvant être considérées comme pratiques de savoir) se voient le plus souvent assignées à n'être que les supports ou les relais de ces processus et de ces logiques d'ordre général. Constat également opéré avec regret dans le contexte anglo-saxon, par Bernstein:

La littérature de la sociologie de l'éducation est considérable; mais si nous passons en revue les textes majeurs qui nous donnent une vue d'ensemble de ses centres 
d'intérêts [...], nous ne trouvons aucune prise en compte systématique des principes de la pratique spécialisée de communication, qui sont pourtant les traits distinctifs de l'activité centrale de l'école, la transmission/acquisition. [...] Qu'est-ce que l'éducation [pour une telle sociologie]? Un relais pour des relations de pouvoir qui lui sont extérieures. L'efficacité spécifique de ce relais importe peu. La communication pédagogique du système éducatif est seulement un relais pour quelque chose d'autre. [...] Que la sociologie de l'éducation se soit rarement tournée vers l'analyse des traits intrinsèques qui définissent en propre la forme spécialisée de communication que réalise le discours pédagogique n'est sans doute pas sans intérêt. Nombre de ses analyses, en particulier celles qui se rattachent aux théories de la reproduction, de la résistance et du changement culturels, se donnent comme allant de soi le discours qui est leur sujet même. Ces théories considèrent, en particulier, que le discours pédagogique sert simplement de support à d'autres voix, celle de la classe, celle du sexe, celle de la race. Elles analysent les discours éducatifs pour leur pouvoir de reproduire les relations entre dominants et dominés, relations qui sont extérieures au discours mais qui pénètrent les relations sociales et les moyens de transmission et d'évaluation du discours pédagogique. On reconnaît souvent qu'il manque une voix au discours pédagogique, celle des classes populaires; mais ce que nous voudrions montrer ici, c'est que ce qui est absent du discours pédagogique dans ce type d'analyse, c'est sa propre voix (Bernstein, 1992, pp. 20-21).

La seconde conséquence, qui n'est pas propre à la sociologie de l'éducation, réside dans le risque et la tentation du relativisme, qui, en l'occurrence, peut conduire nombre de travaux ou de chercheurs à penser ou à donner à penser que l'on pourrait rendre compte des processus de production de «l'échec», de la sélection et des inégalités scolaires en excluant ou en marginalisant la question de la transmission et de l'appropriation des savoirs, en minimisant ou en déniant, de fait, toute composante et réalité cognitive à ces processus de production considérés et étudiés comme s'ils ne relevaient, pour l'essentiel, que de l'affrontement, plus ou moins conflictuel, des points de vue et microperspectives ou des habitus des protagonistes, des logiques d'action ou de distinction, des stratégies de survie et de préservation de soi qu'ils mettent en œuvre, ou de «simples» phénomènes d'étiquetage et de catégorisation, et sans que soit prise en considération la question des rapports entre ces différents phénomènes et l'affrontement à des contenus et des pratiques de savoir dont les normes et les critères de pertinence et d'efficacité ne leur sont pas réductibles. Au-delà de l'intérêt et de la pertinence de tels travaux et de leur apport réel à la mise à jour de phénomènes méconnus ou minorés, on peut néanmoins se demander si une telle posture de déspécification de l'institution scolaire n'est pas aujourd'hui devenue un obstacle puissant pour mieux comprendre et mieux agir contre les inégalités. Les visions et les descriptions "dé-scolarisées» (Lahire, 1999) de l'univers et de l'expérience scolaires ainsi produites, les avis et recommandations dont elles s'accompagnent parfois à l'adresse des acteurs et des décideurs des politiques éducatives semblent bien souvent accréditer ou même appeler de leurs vœux une évolution en termes de 
désinstitutionnalisation de l'École 3 qui ne penserait possibles et souhaitables que les visées de redéfinition du "sens» de l'expérience scolaire des élèves ou des pratiques professionnelles des maitres portant prioritairement sur le cadre de l'établissement et sur les relations de personnes (y compris le rapport du sujet-élève à lui-même), c'est-à-dire se situant en dehors ou en marge des activités d'enseignement-apprentissage et des rapports au savoir et à l'étude qui s'y importent, s'y confrontent et s'y mettent à l'épreuve. Problématique qui, à mon sens, campe sur un clivage théorique et une opposition très discutables entre apprentissages et socialisation ou subjectivation, et qui risque d'aller beaucoup moins dans le sens de la démocratisation de l'accès au savoir et à son exercice critique que dans celui d'une fragmentation sociale accrue du système éducatif entre, d'un côté, des établissements où les apprentissages et la culture participeraient du développement personnel des enfants d'origine favorisée et, de l'autre, des établissements où on viserait à socialiser et à "consoler les pauvres» (Goffman, 1989) plus qu'à leur fournir les instruments intellectuels de leur émancipation, où on tenterait de répondre à une absence ou une perte de sens des apprentissages et de leurs contenus par la promotion ou le développement d'une sociabilité et d'une convivialité sans objets.

\section{Pour des rapports renouvelés entre sociologie, psychologie et didactique}

Mais sans doute convient-il d'inverser le questionnement, et de se demander si, aux versions déscolarisées de l'univers scolaire qu'offrent nombre de travaux sociologiques, ne répondent pas une certaine asepsie sociale de la réflexion, de la recherche et de la pratique pédagogiques, une tendance plus ou moins affirmée à ignorer ou à minorer les questions posées par la sociologie des inégalités scolaires, par exemple, celle que posait il y a 15 ans Philippe Perrenoud lorsqu'il interrogeait les effets sociaux des "pédagogies nouvelles» (Perrenoud, 1995). Il me semble que l'on pourrait tenir encore aujourd'hui les propos que tenait JeanClaude Passeron en 1989:

Les sciences sociales sont, dans la morphologie des communautés savantes travaillant sur l'enseignement, sinon les parentes pauvres $d u$ moins les parentes lointaines et un peu oubliées de la réflexion et de la pratique pédagogiques. [...] La forte liaison, pour ne pas dire le mariage, qu'a nouée dès le XVIII siècle le pédagogue (praticien ou théoricien) avec la réflexion psychologique (et plus tard avec la recherche psychologique) tend aujourd'hui encore [...] à maintenir le sociologue (ou l'historien) dans le rôle périphérique du cousin indésirable ou du rôdeur douteux» (p. 354).

C'est l'idée même d'une possible pédagogie générale, fût-elle différenciée ou individualisée, ou de préceptes pédagogiques généraux, potentiellement indépendants des savoirs et des champs disciplinaires et conceptuels et de leur construc- 
tion historique, et des contextes sociaux d'enseignement et d'éducation qui est interrogée par de tels propos, ainsi que la pertinence, au regard des enjeux affirmés d'efficacité et de démocratisation des apprentissages, d'une conception de l'élève ou de l'apprenant comme être générique, abstrait, universel, ou comme pur sujet cognitif ou épistémique. N'est-ce pas également le risque d'un partage du travail entre une sociologie des inégalités scolaires insuffisamment soucieuse des modalités effectives des activités de transmission et d'appropriation des savoirs et techniques intellectuelles, et une recherche en pédagogie ou en psychologie des apprentissages, insuffisamment soucieuse des différents contextes sociaux, institutionnels et didactiques dans lesquels sont toujours situées ces activités, partage du travail qui me semble dommageable à une meilleure intelligibilité des processus de production de l'échec et des inégalités scolaires?

Travailler à dépasser un tel partage du travail requiert sans doute de pouvoir établir des rapports renouvelés entre sociologie, psychologie et didactique (cf. pour un dialogue entre didactique et sociologie, Johsua et Lahire, 1999). Entreprise qui pose bien sûr de redoutables problèmes épistémologiques (chacun des champs disciplinaires étant dès lors convoqué non seulement pour ses apports et questionnement spécifiques, mais aussi en ses limites et en ses débats internes), méthodologiques (concernant en particulier les modalités et possibilités d'interprétation, de validation et de généralisation des données recueillies et des processus analysés) et conceptuels, que nous sommes bien loin d'avoir résolus, ni même inventoriés. Un des enjeux me semble être notre capacité collective à élaborer et à mettre à l'épreuve des catégories interprétatives qui facilitent le dialogue entre approches quantitatives et factorielles et approches processuelles, cliniques ou dialogiques (Grossen, 1999) ainsi que les échanges et questionnements mutuels entre disciplines.

De telles catégories ne sont pas légion. Parmi celles-ci, celle de malentendu, dont Passeron rappelle qu'elle a présidé aux premières enquêtes qu'il a menées avec Bourdieu sur le fonctionnement et les dysfonctionnements de l'enseignement supérieur français, et qui leur a permis de mettre à jour «l'entente perverse entre les attentes des professeurs et celles des étudiants» (Passeron, 1989), me semble avoir toujours un pouvoir heuristique important pour mieux comprendre les modalités de production de l'échec et des inégalités scolaires, pour mieux identifier les processus, individuels et sociaux, institutionnels et didactiques qui facilitent ou, au contraire, gênent, voire interdisent, l'appropriation des savoirs par des élèves appartenant à différents milieux sociaux. Elle est une de celles qui paraît être le mieux à même de permettre d'appréhender l'École et la scolarisation non plus seulement comme cadre ou comme scène où se révèlent ou se construisent des logiques et des processus sociaux extérieurs et d'ordre général, mais également comme lieu et creuset d'une activité et de processus spécifiques engageant différentes catégories d' "acteurs» et différents niveaux de détermination.

Cette notion converge avec celle de contrat didactique élaborée et mise en œuvre dans les recherches en didactique (Johsua, 1988; Schubauer-Leoni, 1988) 
pour mettre à jour, d'une part, la nécessité, afin que la transmission-acquisition de savoirs puisse avoir lieu, d'une base interprétative commune et d'un minimum d'intercompréhension mutuelle entre les différents protagonistes impliqués (Mercer, 1995), mais aussi, d'autre part, les risques de méprise et de méconnaissance, et donc de rupture de contrat, dus au caractère asymétrique et implicite de ses clauses, à leur nécessaire réorganisation au fur et à mesure de l'avancée du temps didactique, et au fait que les présupposés sur lesquels il repose sont très inégalement partagés par les élèves, selon leurs expériences et leurs conditions de socialisation antérieures et extérieures à l'École. La notion de malentendu permet ainsi d'aller au delà de celle de métier d'élève (Perrenoud, 1984; Sirota, 1993) en interrogeant non seulement la conformité institutionnelle mais également la pertinence cognitive des modes d'interprétation et de description par les élèves des exigences, des situations et des activités d'apprentissage auxquelles ils sont confrontés. Différents travaux (Barrère, 1997; Bautier et Rochex, 1997, 1998; Cèbe, 2000; Charlot et al., 1992; Lahire, 1993) semblent ainsi permettre d'affirmer que tout ne se vaut pas de ce point de vue et de repérer des modes de rapports au savoir, au travail et au langage contrastés et étroitement liés à la difficulté ou à la réussite scolaires. Ainsi, au premier de ces deux pôles, l'opacité et les malentendus qui pèsent sur l'interprétation des situations et activités scolaires conduisent fréquemment les élèves à ne pas ou à ne guère pouvoir percevoir celles-ci autrement que comme succession ou juxtaposition hétéroclite de tâches et d'exercices parcellaires, d'obligations formalistes et de rituels, dont la signification s'épuise dans leur effectuation ou leur observance, et qu'ils ne peuvent parler ou décrire autrement que de manière extérieure à ce qui en fait la logique propre au-delà de leur forme strictement scolaire, comme si, dans une logique étroitement behavioriste, il suffisait d'effectuer les tâches et de se conformer aux rituels pour que l'apprentissage s'effectue. Malentendus et opacité quant à la nature du travail intellectuel requis pour apprendre qui vont souvent de pair avec ce qu'Anne Barrère décrit chez les lycéens comme croyance en «l'équivalenttravail», c'est-à-dire en un postulat de transparence et de proportionnalité de la réussite par rapport à la quantité de travail fourni (pour réussir, il faut et il suffit de travailler, et l'on doit réussir d'autant mieux que l'on travaille plus). Ainsi trouve-t-on, aux différents niveaux du système éducatif, nombre d'élèves mobilisés voire très mobilisés pour réussir à l'école tout en demeurant peu efficaces du point de vue de l'appropriation des savoirs. Croyant faire ce qu'il est requis en s'acquittant des tâches et en se conformant aux prescriptions scolaires, sans pour autant être à même de mobiliser l'activité intellectuelle pertinente pour un réel travail d'acculturation, ils estiment en être quitte avec les réquisits de l'institution, et satisfaire ainsi aux conditions de la réussite. Est-il nécessaire de préciser que, si tel est rarement le cas, désillusion et sentiment d'injustice, rancœur et ressentiment sont, eux, bien souvent au rendez-vous ? À l'autre pôle, celui de la réussite et de l'appropriation effective des savoirs et des modes de travail intellectuel, les élèves perçoivent peu ou prou qu'il existe une unité et une spécificité 
des savoirs et des domaines d'apprentissage qui transcende la succession et la juxtaposition des tâches et exercices. Ils peuvent dès lors donner à leur activité un sens cognitif et culturel qui transcende la nécessité de s'acquitter de tâches morcelées, de routines ou d'exigences comportementales ou disciplinaires. Leurs conduites en classe et les propos par lesquels ils les commentent font preuve d'une autonomie relative par rapport au travail, aux consignes et à la personne de l'enseignant et par rapport aux rituels de la classe. S'ils ne sont pas nécessairement plus disciplinés ou plus travailleurs que leurs pairs plus en difficulté, l'activité qu'ils parviennent à mettre en ouvre dans et hors la classe apparaît en revanche plus pertinente.

Reste que l'on se saurait chercher unilatéralement la source des malentendus du seul côté des élèves et de leurs familles sans interroger ce que leur création, leur récurrence, voire leur renforcement, doivent aux pratiques professionnelles des agents du système éducatif, aux modes de définition et de transmission de la culture scolaire et aux modes de fonctionnement de l'institution. La notion de malentendu vient alors interroger le rapport entre, d'une part, le souci affirmé par la majorité des enseignants et autres professionnels du système éducatif de faciliter la réussite des élèves et d'œuvrer à la démocratisation de l'accès au savoir, et, d'autre part, leurs modes de faire et de penser face à des élèves concrets, et tout particulièrement la façon dont ils interprètent et traitent les difficultés que ceux-ci peuvent éprouver, et dont ils tentent de «s'adapter» aux élèves qui sont les moins à même d'interpréter de manière pertinente les situations scolaires et les exigences du contrat didactique, les moins familiarisés avec l'univers scolaire et ses modes de fonctionnement. Elle permet également d'interroger le rapport entre, d'une part, les mesures structurelles, les prescriptions et incitations officielles, leurs présupposés et objectifs proclamés, leurs modalités d'élaboration et de mise en œuvre et, d'autre part ce que l'on peut percevoir et évaluer de leurs effets sociaux et institutionnels.

Concernant le premier de ces deux registres, différents travaux, portant par exemple sur la mise en œuvre de la politique de Zones d'éducation prioritaire (Bouveau et Rochex, 1997; Chauveau et Rogovas-Chauveau, 1995; Glasman 1992) montrent qu'il ne suffit pas "d'innover», ni de vouloir adapter les modes de travail pédagogique aux élèves en difficulté pour, ipso facto, réduire ou lever les malentendus d'ordre sociocognitif entre ces élèves, les enseignants et les exigences propres à l'appropriation des savoirs et au travail intellectuel, et que les conceptions pédagogiques et les pratiques dites innovantes qui font aujourd'hui l'objet de fortes incitations institutionnelles peuvent, autant que les conceptions et pratiques dites traditionnelles, méconnaître, entériner voire renforcer ces malentendus. Si les constats et analyses ainsi opérés ne sont pas aisément généralisables, ils convergent avec les préoccupations et les travaux et résultats plus anciens de Viviane Isambert-Jamati (1984, 1990), de Philippe Perrenoud (1995), ou d'Éric Plaisance (1986), non seulement pour interroger les enjeux sociaux des contenus d'enseignement et de leurs modes d'élaboration et de transmission, 
mais aussi pour dire combien il est nécessaire, pour ce faire, de se déprendre des modes de catégorisation des pratiques professionnelles enseignantes les plus courants. Aux modes de catégorisation dichotomiques profanes ou institutionnels innovation $v$ s tradition, pédagogie active ou différenciée $v s$ transmissive ou frontale, centration sur l'élève ou l'enfant vs sur les disciplines... -, voire à certaines typologies savantes des enseignants et de leur travail (sur ce type d'études typologiques, cf. Hédoux, 1988), on préférera les interrogations et les études en termes de pédagogies rationnelles, explicites vs implicites (Bourdieu et Passeron 1970) ou visibles $v$ invisibles (Bernstein, 1975), sans penser pour autant que les clauses du contrat didactique puissent être jamais transparentes, l'enjeu étant néanmoins de mieux en percevoir, pour travailler à les réduire, les composantes socialement implicites et opaques.

Ce sont des préoccupations voisines, interrogeant les pratiques enseignantes quant à leurs modalités de traitement des contenus d'enseignement et quant à leurs modalités d'ajustement aux activités et difficultés des élèves, qui conduisent Roland Goigoux à une relecture critique des travaux sur l'effet-maître. Dans ses propres recherches portant sur les pratiques d'enseignement de la lecture à l'école primaire et dans l'enseignement adapté, il montre que ces modalités d'ajustement s'avèrent plus ou moins pertinentes: si certaines constituent réellement une aide bénéfique aux élèves, d'autres se révèlent peu efficaces, voire contre-productives, parce qu'elles échouent à se situer sur un registre cognitif et/ou parce qu'elles entérinent ou même renforcent les malentendus qui pèsent sur l'activité des élèves (Goigoux, 2000, 2001). Ces constats et analyses le conduisent à déplorer que «le bilan des travaux sur "l'effet-maître" reste très lacunaire sur le plan de la caractérisation des pratiques efficaces", et à attribuer cette faiblesse au fait que les catégorisations et les «variables» communément utilisées pour évaluer l'efficacité différentielle des pratiques, appréhendant celles-ci à partir de méthodologies la plupart du temps déclaratives et selon des catégories trop grossières pour élucider les modes de faire réellement mis en œuvre, ne s'avèrent pas ou guère pertinentes pour trouver des éléments de réponse à la question posée, parce qu'elles négligent la nature des enjeux de savoir dans l'étude des pratiques et des interactions scolaires effectives. Goigoux propose également de compléter l'approche didactique "classique» des pratiques enseignantes par une approche de type ergonomique qui s'efforce de mettre à jour et de prendre en considération dans l'analyse les ressources dont disposent les maîtres et les contraintes qui pèsent sur leur activité professionnelle. Il soutient que toute visée ou entreprise de transformation des pratiques professionnelles qui ne prendrait pas suffisamment en considération les contraintes de situation qui pèsent sur elles et qui ne se préoccuperait pas suffisamment des conditions concrètes de possibilité de la réorganisation souhaitée des conceptions et modes de faire, serait condamnée à l'échec ou à la production d'effets indésirables voire contraires aux objectifs poursuivis.

Ce souci d'interroger les conditions de la transformation rejoint celui que manifeste Sylvain Broccolichi à partir de ses travaux portant sur les interruptions 
précoces d'études et sur les jeunes sortant du système éducatif sans qualification, ou sur les effets de leurre, les déconvenues et désillusions que produisent, pour nombre d'élèves et de familles de milieux populaires, les effets conjugués d'une politique volontariste de massification de l'enseignement secondaire, plus soucieuse de gestion des flux d'orientation que de réelle amélioration des conditions d'apprentissage, et d'une mise en concurrence croissante des établissements secondaires (Broccolichi, 1995; Broccolichi et Larguèze, 1996; Broccolichi et Euvrard, 1993). Ces travaux montrent combien les politiques scolaires et les mesures volontaristes visant au maintien dans l'enseignement secondaire des élèves en difficulté qui en étaient autrefois exclus, sans pour autant être à même de mettre en œuvre les conditions d'une action éducative efficace pour les élèves qui dépendent le plus de l'École pour acquérir ce qu'elle requiert, ne pouvaient que faire surgir des difficultés de tous ordres, propres à susciter rancœurs et ressentiments chez ces élèves et leurs familles, et à détériorer notablement les conditions de travail des enseignants, au risque de créer des situations particulièrement explosives dans les établissements où les difficultés sociales et scolaires sont les plus concentrées. Ils montrent également combien la méconnaissance des conditions de transformation démocratique de notre système éducatif (ou leur mépris) conduit nombre de discours institutionnels ou même savants sur les enseignants et leurs pratiques à interpréter en termes de résistance au changement ce qui devrait d'abord être analysé comme symptôme d'une transformation inconséquente ou paradoxale du système éducatif, dont les présupposés, explicites ou impensés, ne peuvent que déboucher sur des incompréhensions, des malentendus et des effets pervers allant à l'encontre des objectifs de démocratisation affichés (Broccolichi et Ben-Ayed, 1999). Où l'on voit que les débats et recherches sur «l'échec» ou la «réussite» scolaires, sur leurs conditions de production et sur les rapports complexes et rien moins qu'évidents entre évolutions et démocratisation de notre système éducatif, ne peuvent pas ne pas rencontrer ceux qui s'intéressent aux politiques éducatives, à leur marge d'autonomie, à leurs modes d'élaboration et de mise en ouvre et à l'appréciation de leurs effets sociaux.

\section{Notes}

1 En revanche, cette période a vu l'inversion des inégalités entre sexes au profit non plus des garçons mais des filles. La diversification des filières semble avoir accéléré l'expansion de la scolarité des filles alors qu'elle contrecarrait celle des garçons, particulièrement dans les milieux populaires. La mise en rapport des inégalités sociales et des inégalités entre sexes mériterait un traitement beaucoup plus approfondi que ce qui est possible dans le cadre de cet article.

2 Parmi lesquelles figurent évidemment les travaux menés et impulsés par Viviane IsambertJamati.

3 Me semblent tout particulièrement s'inscrire dans une telle perspective les travaux de Ballion (1993) et ceux de Dubet (Dubet, 1991, Dubet et Martucelli, 1996), que nous avons discutés dans Bautier et Rochex, 1998, et, plus systématiquement, dans Rochex 2000. 


\section{Références bibliographiques}

Baktavatsalou, R. \& Pons, C. (1998). Comparaison des performances en lecture-compréhension des élèves en fin de CM 2 à dix ans d'intervalle (1987-1997) (Note d'information, 98-39). Paris: Direction de la programmation et du développement.

Ballion, R. (1991). La bonne école. Évaluation et choix du collège et du lycée. Paris: Hatier.

Ballion, R. (1993). Le lycée, une cité à construire. Paris: Hachette-Éducation.

Barrère, A. (1997). Les lycéens au travail. Paris: PUF, 1997.

Barrère, A. \& Sembel, N. (1998). Sociologie de l'éducation. Paris: Nathan.

Baudelot, Ch. \& Establet, R. (1989). Le niveau monte. Paris: Seuil.

Bautier, E. \& Rochex, J.-Y. (1997). Apprendre: ces malentendus qui font la différence. In J.P. Terrail (Ed.), La scolarisation de la France. Critique de l'état des lieux. Paris: La Dispute.

Bautier, E. \& Rochex, J.-Y. (1998). L'expérience scolaire des nouveaux lycéens. Démocratisation ou massification? Paris: Armand Colin.

Beaud, S. \& Pialoux, M. (1999). Retour sur la condition ouvrière. Paris. Fayard.

Bernstein, B. (1975). Classes et pédagogies: visibles et invisibles. Paris: CERI-OCDE.

Bernstein, B. (1992). La construction du discours pédagogique et les modalités de sa pratique. Critiques sociales, 3-4, 20-58.

Bourdieu, P. \& Passeron, J.-C. (1970). La reproduction. Eléments pour une théorie du système d'enseignement. Paris: Minuit.

Bouveau, P. \& Rochex, J.-Y. (1997). Les ZEP, entre École et société. Paris: CNDP-Hachette.

Broccolichi, S. (1995). Orientations et ségrégations nouvelles dans l'enseignement secondaire. Sociétés contemporaines, 21, 15-27.

Broccolichi, S. \& Ben-Ayed, C. (1999). L'institution scolaire et la réussite de tous aujourd'hui: "pourrait mieux faire". Revue française de pédagogie, 129, 39-51.

Broccolichi, S. \& Larguèze, B. (1996). Les sorties sans qualification du système éducatif moins de cinq ans après l'entrée au collège. Éducation et formations, 46, 81-102.

Broccolichi, S. \& Oeuvrard, F. (1993). L'engrenage. In P. Bourdieu (Ed.), La misère du monde. Paris: Seuil.

Broccolichi, S. \& Van Zanten, A. (1997). Espaces de concurrence et circuits de scolarisation. L'évitement des collèges publics d'un district de la banlieue parisienne. Annales de la Recherche Urbaine, 75, 5-31.

Cèbe, S. (2000). Développer la conceptualisation et la prise de conscience à l'école maternelle: effets sur l'efficience scolaire ultérieure $d u C P$ au $C E 2$. Une contribution à la prévention de l'échec scolaire des élèves de milieux populaires. Thèse de Doctorat en psychologie, Aix-Marseille, Université de Provence.

Charlot, B., Bautier, E. \& Rochex, J.-Y. (1992). École et savoir dans les banlieues... et ailleurs. Paris: Armand Colin..

Chauveau, G. \& Rogovas-Chauveau, E. (1995). À l'école des banlieues. Paris: ESF.

Dubet, F. (1991). Les lycéens. Paris: Seuil.

Dubet, F. \& Martucelli, D. (1996). À l'école. Paris. Seuil.

Duru-Bellat, M. (2000). L'évolution des problématiques et des méthodologies dans l'analyse des différenciations sociales de carrières scolaires», In M. Froment, M. Caillot \& M. Roger (Ed.), 30 ans de sciences de l'éducation à Paris V. Paris: PUF.

Duru-Bellat, M. \& Kieffer, A. (1999). La démocratisation de l'enseignement "revisitée". Une mise en perspective historique et internationale des inégalités des chances scolaires en France (Les Cahiers de l'IREDU). Dijon: Université de Bourgogne.

Duru-Bellat, M. \& Van Zanten, A. (1999). Sociologie de l'école (2e édition). Paris: Armand Colin.

Les sortants du système scolaire sans qualification (2000). Éducation et formations, 57.

Forquin, J.-C. (1989). École et culture. Le point de vue des sociologues britanniques. Bruxelles: De Bøeck. 
Galland, O. (1988). Représentations du devenir et reproduction sociale: le cas des lycéens d'Elbeuf. Sociologie du travail, 3, 399-417.

Glasman, D. (1992). L'école réinventée ? Le partenariat dans les zones d'éducation prioritaires. Paris: L'Harmattan.

Goffman, E. (1989). Calmer le jobard: quelques aspects de l'adaptation à l'échec. In collectif. Le parler frais d'Erving Goffman. Paris: Minuit.

Goigoux, R. (2000). Les élèves en grande difficulté de lecture et les enseignements adaptés (Rapport d'étude pour la Direction des enseignements scolaires). Suresnes: Éditions du CNEFEI.

Goigoux, R. (2001). Enseigner la lecture à l'école primaire. Note de synthèse pour l'Habilitation à Diriger des Recherches, Université Paris VIII.

Grossen, M. (1999). Approche dialogique des processus de transmission-acquisition de savoirs: une brève introduction. Actualités psychologiques, 7, Institut de Psychologie, Université de Lausanne, 1-32.

Hédoux, J. (1988). Les enseignants du second degré en France: études typologiques. Recherche et Formation, 4, 37-50.

Henriot-Van Zanten, A., Derouet, J.-L. \& Sirota, R. (1987a). Approches ethnographiques en sociologie de l'éducation: l'école et la communauté, l'établissement scolaire, la classe (1ère partie). Revue française de pédagogie, 78, 73-108.

Henriot-Van Zanten, A., Derouet, J.-L. \& Sirota, R. (1987b). Approches ethnographiques en sociologie de l'éducation: l'école et la communauté, l'établissement scolaire, la classe (2è partie). Revue française de pédagogie, 80, 69-97.

Isambert-Jamati, V. (1984). (Culture technique et critique sociale à l'école élémentaire. Paris: PUF.

Isambert-Jamati, V. (1985). Quelques rappels de l'émergence de l'échec scolaire comme problème social dans les milieux pédagogiques français. In E. Plaisance (Ed), L'échec scolaire, nouveaux débats, nouvelles approches sociologiques. Paris: Éditions du CNRS.

Isambert-Jamati, V. (1990). Les savoirs scolaires. Enjeux sociaux des contenus d'enseignement et de leurs réformes. Paris. Éditions universitaires.

Johsua, S. (1988). Le «contrat didactique» et l'analyse des phénomènes didactiques. Interactions didactiques, 9, Université de Neuchâtel, 35-44.

Johsua, S. \& Lahire, B. (1999). Pour une didactique sociologique. Éducation et sociétés, 4, 29-56.

Lahire, B. (1993). Culture écrite et inégalités scolaires. Lyon: PUL.

Lahire, B. (1999). La sociologie de l'éducation et l'opacité des savoirs. Éducation et sociétés, 4, 15-28.

Mercer, N. (1995). The guided construction of knowledge. Talk amongst teachers and learners. Clevedon: Multilingual Matters.

Merle, P. (2000). Le concept de démocratisation de l'institution scolaire: une typologie et sa mise à l'épreuve. Population, 55(1), 15-50.

Montandon, C. \& Perrenoud, Ph. (1987). Entre parents et enseignants: un dialogue impossible? Berne, Peter Lang.

Passeron, J.-C. (1991). Le raisonnement sociologique. L'espace non-poppérien du raisonnement naturel (Les trois savoirs sur le savoir. Sociologie et sciences de l'éducation, pp. 347-354). Paris: Nathan. (Republié de Actes du colloque Finalités des enseignements scientifiques, Marseille, CCSTI, 1989).

Perrenoud, Ph. (1984). La fabrication de l'excellence scolaire. Genève: Droz.

Perrenoud, Ph. (1995). La pédagogie à l'école des différences. Fragments d'une sociologie de l'échec (Les pédagogies nouvelles sont-elles élitaires? Réflexion sur les contradictions de l'école active, pp. 105-118). Paris: ESF. (Republié de Actes du colloque Classes populaires et pédagogie, Université de Rouen, 1985).

Plaisance, E. (1986). L'enfant, la maternelle, la société. Paris: PUF.

Poncet, P. (2000). Les facteurs de réussite au début du collège d'après les panels d'élèves entrés en sixième en 1989 et 1995 (Note d'information 00-54). Paris: Direction de la programmation et du développement. 
Prost, A. (1986). L'enseignement s'est-il démocratisé ? Paris: PUF.

Prost, A. (2001). La démocratisation de l'enseignement en France depuis la Seconde Guerre mondiale. Revue suisse des sciences de l'éducation, 1, 73-92.

Queiroz (De), J.-M. (1981). La désorientation scolaire. Sur le rapport social de familles populaires à la scolarisation. Thèse de Doctorat en sociologie, Université Paris VIII.

Queiroz (De), J.-M. (1991). Les familles et l'école. In F. De Singly (Ed), Familles: l'état des savoirs. Paris, La Découverte.

Queiroz (De), J.-M. (1995). L'école et ses sociologies. Paris, Nathan.

Rayou, P. (1998). La Cité des lycéens. Paris: L'Harmattan.

Rochex, J.-Y. (1991). L'implosion scolaire. In J.-P. Durand \& F.-X. Merrien (Ed.). Sortie de siècle. La France en mutation. Paris: Vigot.

Rochex, J.-Y. (1993). Normes et normativité en sociologie de l'éducation. Futur Antérieur, 1920, 195-206.

Rochex, J.-Y. (1995). Le sens de l'expérience scolaire. Paris: PUF.

Rochex, J.-Y. (2000). Expérience scolaire et procès de subjectivation. L'élève et ses milieux. Note de synthèse pour l'Habilitation à Diriger des Recherches, Université Paris VIII.

Schubauer-Leoni, M.-L. (1988). Le contrat didactique dans une approche psycho-sociale des situations d'enseignement. Interactions didactiques, 9, Université de Neuchâtel, 63-75.

Sirota, R. (1993). Le métier d'élève. Revue française de pédagogie, 104, 85-108.

Terrail, J.-P. (Ed.) (1997). La scolarisation de la France. Critique de l'état des lieux. Paris: La Dispute.

Thin, D. (1998). Quartiers populaires. L'école et les familles. Lyon: PUL.

\section{Schulversagen und Demokratisierung: Fragen, Realitäten, Konzepte, Probleme und Forschungsresultate}

\section{Zusammenfassung}

Die Begriffe des Schulversagens und der Demokratisierung des Bildungssystems bzw. der Zugänge zum Wissen sind eng miteinander verbunden, und gleichzeitig sind sie in ihrem gegenseitigen Verhältnis eminent problematisch. Mehrdeutig, bezeichnen sie widersprüchliche Realitäten, die in der Analyse unterschieden werden müssen, dabei aber viel von ihrer Gegensätzlichkeit verlieren. Besser verstehen, um besser zu agieren, verlangt deshalb, das Risiko einer Arbeitsteilung zu überwinden zwischen einer Soziologie der ungleichen Bildungschancen, die sich zu wenig um die effektive Art und Weise der Vermittlung und Aneignung von Wissen und intellektuellen Techniken kümmert, und einer pädagogischen und lernpsychologischen Forschung, welche die unterschiedlichen sozialen, institutionellen und didaktischen Kontexte, in denen ihre Aktivitäten immer situiert sind, zu wenig beachtet. Dies würde verlangen, dass wir neue Beziehungen zwischen Soziologie, Psychologie und Didaktik aufzubauen vermöchten. 
Insuccesso scolastico e democratizzazione: sfide, realtà, concetti, problemi e risultati della ricerca

\section{Riassunto}

Le nozioni di insuccesso scolastico, di democratizzazione del sistema educativo e di accesso al sapere/ai saperi sono al tempo stesso strettamente legate e molto problematiche. Dai significati molteplici, esse designano delle realtà contradditorie che occorre distinguere a livello di analisi. L'obiettivo di capire meglio per poter agire meglio richiede un lavoro teso a superare il rischio di una divisione del lavoro tra, da un lato, una sociologia delle disparità scolastiche preoccupata delle modalità effettive di trasmissione e appropriazione del sapere e delle tecniche intellettuali e, dall'altro lato, una ricerca in psicologia e pedagogia insufficientemente attenta alle differenze legate ai contesti sociale, istituzionale e didattico a cui sono sempre legate le attività di apprendimento. Tutto ciò ci richiede di rinnovare i rapporti tra sociologia, psicologia e didattica.

\section{School Failure and democracy: Questions, realities, concepts, problems and research results}

\section{Summary}

The concept of school failure and the democratization of the school system, or, in other words, the democratic access to knowledge, are closely related. And yet this relationship is eminently problematic because of their contradictory natures. At their core multifaceted and indicative of conflicting realities, they lose many of their contradictions in analysis. In order to better understand this relationship (and thereby respond more appropriately) we need to overcome the risky separation we make between the sociology of unequal educational opportunities and research on teaching and learning. While the one does not pay enough attention to the effective transmission and acquisition of knowledge and learning techniques, the other pays too little attention to the various social, institutional and instructional contexts in which learning is situated. This would mean that we need to establish new relationships between sociology, psychology and teaching methodology. 